\title{
Conclusions de la Journée de Rennes de la Société Française de Filtration
}

\author{
par \\ J. MILLET
}

Au cours de cette journée, nous avons examiné les différents aspects de l'ultrafiltration.

D'abord les aspects scientifiques et techniques de l'utilisation des membranes pour les vins (piquettes, vins de consommation courante, beaujolais) et en matière de laiterie.

Les différentes qualités de filtration ont été étudiées : microfiltration, ultrafiltration, osmose inverse.

Une innovation a été décrite : les membranes minérales de microfiltration.

Des conférenciers ont donné des études économiques permettant de comparer les coûts des procédés de séparation utilisant les membranes et les autres procédés.

Enfin les problèmes de réglementation ont été discutés et les participants ont souhaité un dialogue avec l'administration à ce sujet.

La séance d'aujourd'hui sera poursuivie demain par celle du Club " Membranes » E.D.F. qui réunit des chercheurs, des utilisateurs, des industriels. Son but est de promouvoir la fabrication de membranes françaises de qualité et d'aider à une utilisation optimale des membranes.

La collaboration qui s'instaure entre la Société Française de Filtration, la Société Européenne d'Etude des Membranes et le Club E.D.F. font bien augurer du développement en France des techniques de séparation par membranes. 